\title{
Devising the guidelines: the techniques of pulmonary nodule localization in uniportal video-assisted thoracic surgery-hybrid operating room in the future
}

\author{
Ze-Rui Zhao", Rainbow W. H. Lau' ${ }^{2}$, Peter S. Y. Yu ${ }^{2}$ Calvin S. H. Ng ${ }^{2}$ \\ ${ }^{1}$ Department of Thoracic Surgery, Sun Yat-sen University Cancer Center, Guangzhou 510060, China; ${ }^{2}$ Division of Cardiothoracic Surgery, \\ Department of Surgery, The Chinese University of Hong Kong, Prince of Wales Hospital, Hong Kong SAR, China \\ Contributions: (I) Conception and design: CS Ng, ZR Zhao; (II) Administrative support: CS Ng, RW Lau; (III) Provision of study materials or \\ patients: CS Ng, PS Yu, RW Lau; (IV) Collection and assembly of data: ZR Zhao, CS Ng; (V) Data analysis and interpretation: ZR Zhao, CS Ng; (VI) \\ Manuscript writing: All authors; (VII) Final approval of manuscript: All authors. \\ Correspondence to: Calvin S. H. Ng, MD, FRCS, FCCP. Associate Professor, Division of Cardiothoracic Surgery, The Chinese University of Hong \\ Kong, Prince of Wales Hospital, Shatin, N.T., Hong Kong SAR, China. Email: calvinng@surgery.cuhk.edu.hk.
}

\begin{abstract}
Pulmonary nodules beneath the pleura can be hard to visualize or palpate, especially during the uniportal thoracoscopic surgery. Conventionally, thoracic surgeons would use adjuvant modalities to localize the lesion preoperatively, of which computed tomography-guided hookwire implantation has been adopted most widely due to its feasibility and high success rate. However, procedure-associated complications such as pneumothorax and wire dislodgement can cause patient discomfort or localization failure. Occasionally more healthy tissue is resected than needed to guarantee the lesion is removed and with an adequate margin. A thoracotomy is necessary for specific scenario. With the development of imaging technology, it is now possible to replace the traditional workflow carried out in the radiology suite by centralizing the hookwire placement and uniportal minimally-invasive pulmonary resection inside the hybrid theater which equipped with advanced imaging devices. Theoretically, the advanced intra-operative imaging-guided techniques help to precisely locate and resection pulmonary lesion in a potentially tissue-sparing and quick paradigm.
\end{abstract}

Keywords: Image-guided; hybrid theater; localization

Submitted Oct 21, 2018. Accepted for publication Jan 18, 2019.

doi: $10.21037 /$ jtd.2019.01.82

View this article at: http://dx.doi.org/10.21037/jtd.2019.01.82

\section{Introduction}

Preoperative adjunctive localization techniques including hookwire/microcoil placement or dye labelling conducted under computed tomography (CT) guidance play essential roles in identifying pulmonary lesions during uniportal video-assisted thoracic surgery (VATS). However, such conventional workflow carried out in the radiology suite may increase procedure-related complication and complexity of care. Cone-beam CT (CBCT) shows promising abilities in visualizing the target lesion and its surrounding surgical anatomy, with an average error of less than $2 \mathrm{~mm}$ (1). The hybrid operation room (OR), which integrates CBCT and the OR, is capable of providing unparalleled real-time imaging of the patient, facilitating both the localization and resection procedure to be done in the same room with a one-stop concept, hence reducing the rate of pneumothorax, metallic marker dislodgement, and dye diffusion. As a result, surgeons could perform excisions precisely and safely, even for multifocal lesions, and spare pulmonary tissues $(2,3)$.

\section{Layout of the hybrid theater}

A hybrid OR implemented a multidisciplinary design and is capable of image-guided diagnostic and minimally invasive procedures. The first comprehensive hybrid suite [Advanced 
Multimodal Image-Guided Operating (AMIGO) suite] designed for general thoracic surgery was described by the Brigham and Women's Hospital in 2013 for image-guided VATS (iVATS) (4). The AMIGO suite has three separate but integrated compartments that incorporate magnetic resonance imaging (MRI), near-infrared imaging, CBCT, and positron emission tomography, providing excellent multidisciplinary radiologic support for a variety of surgical applications. In general, the hybrid OR is equipped with at least one of the following structures such as fixed C-Arms, CT or MRI scanners.

A relatively smaller suite was established in our hospital (5). This suite also implements a multidisciplinary design and is capable of image-guided electromagnetic navigation bronchoscopy (ENB) and VATS procedures $(5,6)$. The floor-mounted CBCT has high flexibility and can be moved from a distant parked position to the surgical field without interfering with the laminar airflow in a short time.

\section{Advantages and disadvantages of CBCT over multidetector CT}

Mobile CBCT can provide sub-millimeter spatial resolution combined with soft tissue visibility even at a low radiation dose ( 4.3 mGy/scan) (1). With an open gantry design, the CBCT system has greater working space, better angulation and rotation, as well as can be incorporated with advanced needle guidance system with artificial intelligence assistance (7). Usually, the settings for the CBCT volume acquisition are a 6-second breath-hold and 200-degree rotation protocol with 0.5 degrees/frame, which would generate a total of 397 projections (8). The acquired volume with a cylindrical field of view (FOV) of $24.72 \mathrm{~cm}$ in diameter and $19.01 \mathrm{~cm}$ in height was reconstructed into a matrix of $512 \times 512 \times 391$ voxels, with a slice thickness of $0.5 \mathrm{~mm}$. A large-volume CBCT protocol for large size patient can be used. The protocol consists of 2 consecutive, translated rotations, where the settings for each rotation were 4.5 seconds and 218-degree rotation protocol with 0.8 degrees/frame, generating a total of 273 projections that gave a volume with a cylindrical field of view of $46.39 \mathrm{~cm}$ in diameter and $18.69 \mathrm{~cm}$ in height, which was reconstructed into a matrix of $512 \times 512$ voxels, and with a slice thickness of $0.91 \mathrm{~mm}$. However, an unexpected collision between the surgical table and the rotating C-arm may occur when the operators want to engage both the target lung lesion and the needle entry site into the interested FOV, especially when dealing patients with large chest cavity. More importantly, this may result in repositioning the patients from the lateral decubitus position into a supine or prone one, which would inevitably increase the procedure duration (9). It should be further highlighted that these figures can be variable depending on the specific imaging system used.

In comparison, patient positioning in the closed sliding gantry-based multidetector CT (MDCT) does not limit by the size of the chest because the FOV is larger than that of CBCT. And multidisciplinary teams familiar with traditional CT-guided localization may find it easier to use MDCT in the hybrid OR. Additionally, the CBCT is believed to produce a higher amount of scatter radiation compared with MDCT, as is proved in a study focus on the abdominal imaging (10). However, CBCT may have several advantages in the following terms as suggested by a previous report (11). On the one hand, the MDCT has a greater chance of transferring the patient within the hybrid OR due to its limited flexibility. On the other hand, the iGuide (Siemens Healthcare AG, Forchheim Germany) navigation software incorporated with CBCT system can automatically determine the best needle path for centesis which would reduce the chance of repeated scans during needle insertion.

With a significantly reduced contrast, the visibility of nodules in deflated lungs during surgery via CBCT can be challenging. The clinical scenario will be evident if the hookwire is dislodged from the parenchyma. In a pre-clinical study by Uneri and colleagues (1), a slightly increased dose of CBCT ( 4.6-11.1 mGy) is sufficient to visualize the lesion in collapsed lung tissue. As a result, clinicians would be aware the potential anatomical characteristics of a lesion in the hybrid OR; for example, whether there is a direct endobronchial route for biopsy, or adjacent vessels to be aware of during metallic material reimplantation (3).

\section{Workflows of image-guided metallic implantation}

As discussed, the hybrid OR provides a platform for simultaneous nodule localization with subsequent lung resection for thoracic surgeons. The image-guided localization procedure can either be conducted under local or general anesthesia according to surgeon's preference $(11,12)$. Theoretically, performing the localization under general anesthesia, especially in the lateral decubitus position, has the advantage of reducing patient's discomfort and shortening the time from hookwire insertion to the start of VATS procedure. However, inserting the hookwire 

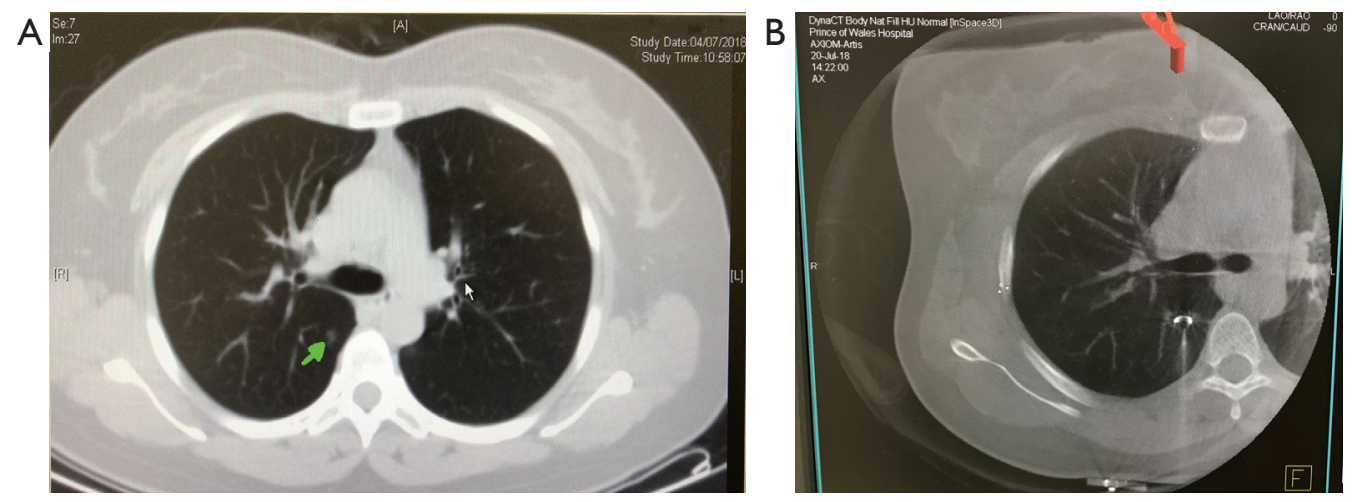

Figure 1 Percutaneous hookwire insertion in hybrid OR. (A) Right upper lobe subcentimeter pure ground glass opacity for uniportal VATS resection marked by green arrow; (B) dynaCT image in hybrid operating room immediately following hookwire insertion. OR, operation room; VATS, video-assisted thoracic surgery.

in the lateral decubitus position may be associated with a higher chance of having trouble in FOV integration in large habitus patients. In some circumstances, the $\mathrm{C}$-arm could collide with the patient (9). Nevertheless, the problem would become less evident if supine or prone position is used for hookwire insertion. Even if a hookwire need to be inserted from the direction of the lateral chest wall, the patient could remain in a supine or prone position in our practice. Besides, in our experience, hookwire insertion under local anesthesia can have a higher chance of localization success because atelectasis can accidentally happen under general anesthesia, which leads to a vague image of the tiny ground-glass lesion within the deflated lung. In addition, it is more comfortable to ask the patient to be positioned prone if such a position is needed for marker insertion.

General workflow demonstration for hookwire insertion and iVATS in hybrid OR:

(I) The patient is positioned appropriately in the supine, lateral decubitus or prone position upon the location of the lesion;

(II) After wrapping the patient's chest and C-arm in sterile covers, a 6 -second end-inspiratory breathhold protocol (6 s Dyna-CT Body, syngo ${ }^{\circledR}$ DynaCT, Siemens Healthcare, Erlangen, Germany) is used to obtain the $3 \mathrm{D}$ real-time image of the thorax (Figure 1A);

(III) The optimal direction and depth of the needle puncture path is determined then laid out by laser cross projecting on the skin of the patient through the syngo Needle Guidance of a syngo X-Workplace;
(IV) Under end-inspiratory breath-hold, the hookwire is deployed and fixed. Real-time fluoroscopic guidance can be used to adjust the orientation and positioning of the virtual needle path (Figure 1B);

(V) The patient is immediately repositioned, undergo general anesthesia with a double-lumen endotracheal tube if the hookwire is placed under local anesthesia, and receive uniportal VATS.

During operation, the target lesion can be estimated by the direction of the hookwire, the relative position of the wire-tip to the target nodule, and the puncture wound on the visceral pleural surface with the distance of the lesion depicted by the DynaCT image. Occasionally, on-table CT imaging can be performed to confirm the accuracy of the hookwire positioning if needed.

\section{Comparison of localization techniques between conventional and hybrid OR}

Though several reports had demonstrated the feasibility of adjuvant localization techniques for pulmonary resection in the hybrid OR $(5,12,13)$, few of them focus on the direct difference between this novel approach against conventional two-stop techniques. In a recent report from our group, 32 pulmonary nodules with a mean size of $9.1 \pm 4.6 \mathrm{~mm}$ were successfully receiving image-guided hookwire localization with local anesthesia and VATS wedge resection under general anesthesia (iVATS) (12). The shortest distance from the lesion to the visceral pleura was $10.8 \pm 8.3 \mathrm{~mm}$ and the depth-to-diameter ratio was $1.5 \pm 1.5$. Another eight patients received standard localization and operation in separate rooms (sVATS) were recruited as a comparison. The results 
showed that patients in the sVATS group experienced a higher chance of wire dislodgement $(25 \%$ vs. $0, \mathrm{P}=0.036)$ and longer "at-risk" period of potential pneumothorax progression as well as dislodgement development (defined as the interval between the completion of hookwire insertion and the beginning of VATS, 109.5 $\pm 57.1 \mathrm{vs}$. $41.1 \pm 15.0$ minutes, $\mathrm{P}=0.011)$ compared with the iVATS group. Interestingly, pneumothorax was found in 15 (47\%) patients immediately after wire insertion on real-time CT scan in the iVATS group. But none of them required additional intervention.

In another similar study conducted by Chao's group, 34 patients with a mean size of $6.45(5.25-7.65) \mathrm{mm}$ and distance to visceral pleura of $7.75(1.5-15) \mathrm{mm}$ underwent iVATS procedure (11). Thirty cases that had similar lesion characteristics were selected as the control group. The authors discovered a significantly reduced "at risk" time in the iVATS (13.06 vs. 215.83 minutes, $\mathrm{P}<0.001)$ than the sVATS group. In contrast, the iVATS approach remarkably increased the time under general anesthesia (163.1 vs. 120.61 minutes, $\mathrm{P}<0.001)$ as well as total $\mathrm{OR}$ utilization time (227.41 vs. 168.68 minutes, $\mathrm{P}<0.001$ ). The researchers also used thermoluminescent dosimeters to quantify the radiation exposure under these two approaches. However, there was no significant difference between iVATS (3.65 $\mathrm{mSv}$ ) and sVATS (6.88 $\mathrm{mSv}, \mathrm{P}=0.506)$ arms.

Actually, with the experience gathered from the iVATS learning curves, surgeons would be able to accomplish most metallic insertion procedure with the patients in lateral decubitus position under general anesthesia (11). Individualized reciprocal positioning protocol of the patient, surgical table, and CBCT helps to reduce the chance of repeated scanning (9). This may partly explain why the average time-at-risk in our report (41.1 minutes) is greater than that of Chao's study (13.06 minutes). In addition, the hookwire insertions in Yu's report (12) were performed under local anesthesia while those in Chao's group were inserted after general anesthesia and intubation. However, it should be noted that the studies mentioned above were not designed in a randomized setting, the choice between sVATS and iVATS should be based on the surgeon's own experience and specific facility.

\section{Future inter-disciplinary combination in hybrid OR: inspirations from ongoing trials}

Though image-guided hookwire iVATS technique works perfectly for peripheral lesions of sub-centimeter size and may decrease the risk of complications associated with implantation, technical limitations inherent from this approach remain as nodules that are located near to the apex, shielded by the scapula and clavicle, at proximity to great vessels or diaphragm can be difficult to reach using the wire. As an advanced bronchoscopic technique, ENB utilizes electromagnetic sensor technology to enable that the tip of the localization guide reaches the proximity of the peripheral lesion during the navigational phase. To decrease the navigational error of ENB technique, we described the use of integrated CBCT and ENB in a hybrid OR to guide and confirm the successful navigation as well as biopsy of an $8-\mathrm{mm}$ lesion located in the right middle lobe (6). It is obvious that the image from the dynaCT in the hybrid OR has superior accuracy over conventional methods like fluoroscopy or endobronchial ultrasound (EBUS) in revealing the direction of the catheter tip. Trans-bronchial injection of dye under ENB guidance also assists localizing pulmonary nodules during VATS sublobar resection. With the help of the hybrid CBCT technique, we are now able to inject triple mixture $(0.2$ to $0.5 \mathrm{~mL})$ of indocyanine green, methylene blue, and standard iohexol imaging contrast in proximity to the target lesions to guide resection margin under uniportal VATS.

General workflow demonstration for ENB dye marking followed by surgery:

(I) The patient is positioned in the supine position under single-lumen endotracheal general anesthesia;

(II) After ENB navigation via the pre-determined route to the peripheral lesion with or without the assistance of fluoroscopy and radial EBUS, the extended working channel is locked in place (Figure 2A). The whole bronchoscopic-extended working channel complex is rested and secured on a non-metallic mount to avoid dislodgement. The dye injection needle is deployed;

(III) DynaCT is performed and the reconstructed image in the hybrid OR shows the relative positions of injection needle and the target lesion (Figure 2B). To avoid interference, the electromagnetic board is disconnected prior to this procedure;

(IV) Once the injection needle is confirmed to approach the lesion, 0.2 to $0.5 \mathrm{~mL}$ methylene blue is injected into the lesion (Figure 2C). If the marked nodule is located more than $10-15 \mathrm{~mm}$ from the visceral pleura (Figure 2D), the needle may be advanced closer to the pleura for a second dose of dye injection (Figure 2E) or the initial dye marking can 

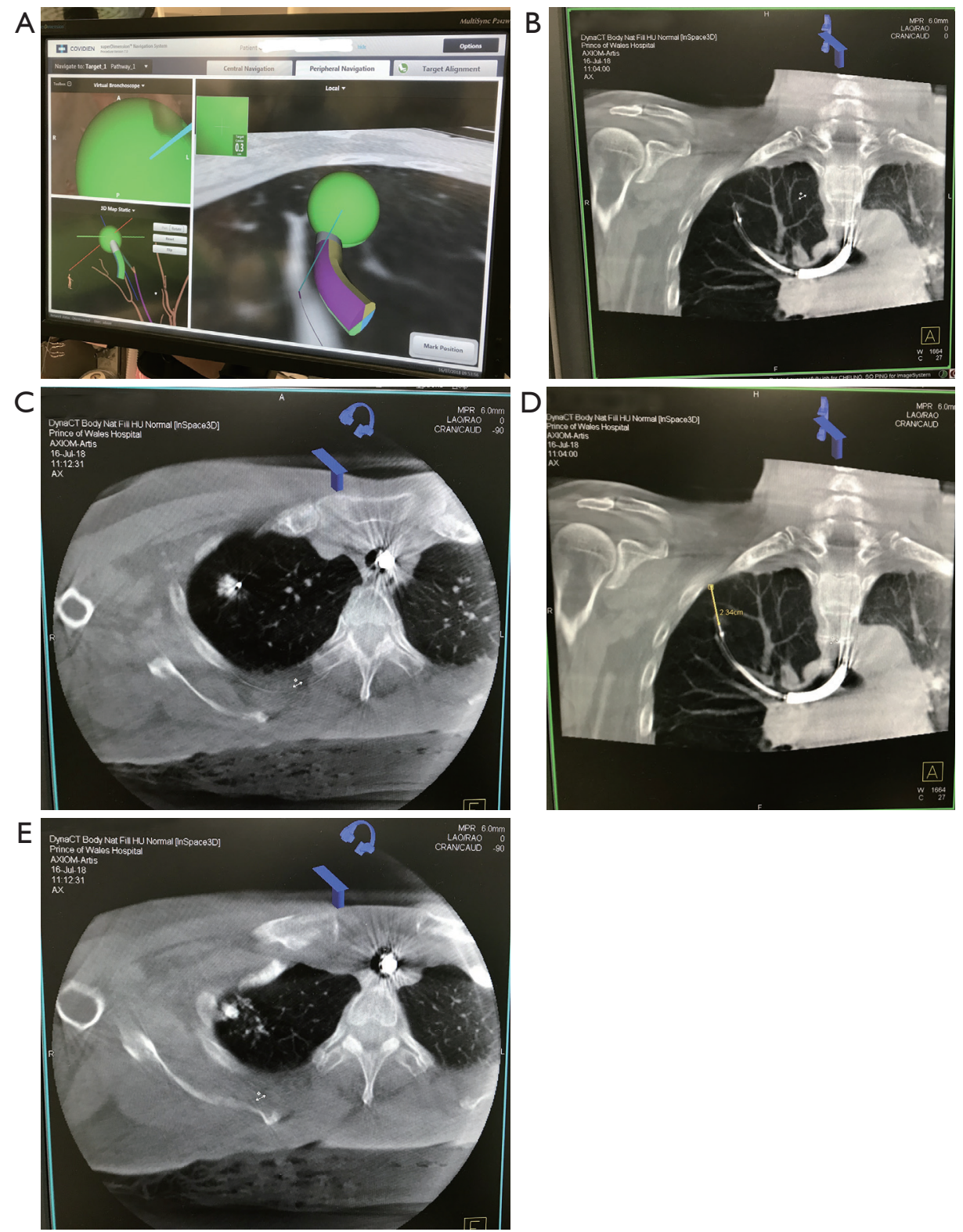

Figure 2 Electromagnetic navigation bronchoscopy dye marking of right upper lobe subcentimeter pure ground glass opacity (GGO) for uniportal VATS resection, (A) navigation of locatable guide to within 3 millimeters from the centre of the target lesion, (B) dynaCT showing the dye injection needle directly on the GGO, (C) dynaCT following injection of mixed iohexol and methylene blue dye contrast at the lung lesion, (D) measurement of distance from target lesion/injection needle to pleura for advancement of needle to pleura for second injection, (E) dynaCT following injection of contrast at the pleura. VATS, video-assisted thoracic surgery.

be performed at midway between the lesion and the nearest pleural surface;

(V) The single-lumen endotracheal tube is replaced with a double-lumen endotracheal tube. The patient is then put in the lateral decubitus position and uniportal VATS performed.
The guided therapeutics surgery program conducted in the Toronto General Hospital also propose several ongoing trials with advanced facilities in the hybrid OR. For example, indocyanine green labelling under nearinfrared fluorescence imaging system for VATS sublobar resection, virtual 4-D electromagnetic tip-tracked 
bronchoscopy system for pulmonary nodule diagnosis and management, and so on (14). No doubt some of these new technologies and trials would allow rapid advancements and improvements in the workflow of different surgical procedures in the near future.

\section{Conclusions}

Centralization of the diagnostic, localizing, and resecting procedures within a specially designed hybrid theater could enhance diagnostic accuracy as well as decrease the risk period of complications developments associated with the implantation of metallic markers. Precise nodule localizing technique could avoid unnecessary excision when treating multiple lesions (15). It is plausible that the hybrid OR may become increasingly important in minimally-invasive surgical management as it establishes a cost-effective, onestop platform for image-guided thoracic surgery.

\section{Acknowledgments}

Funding: This work was supported by Research Grants Council (RGC) General Research Fund (GRF) (14117715).

\section{Footnote}

Conflicts of Interest: CS Ng has an electromagnetic navigation bronchoscopy system SuperDimension Version 7 on loan from Medtronic. The other authors have no conflicts of interest to declare.

\section{References}

1. Uneri A, Nithiananthan S, Schafer S, et al. Deformable registration of the inflated and deflated lung in conebeam CT-guided thoracic surgery: initial investigation of a combined model- and image-driven approach. Med Phys 2013;40:017501.

2. Zhao ZR, Lau RWH, Ng CSH. Hybrid theater and uniportal video-assisted thoracic surgery: the perfect match for lung nodule localization. Thorac Surg Clin 2017;27:347-55.

3. Zhao ZR, Lau RW, Yu PS, Wong RH, Ng CS. Imageguided localization of small lung nodules in video-assisted thoracic surgery. J Thorac Dis 2016;8:S731-S737.

4. Advanced Multimodality Image Guided Operating (AMIGO). Available online: http://www. brighamandwomens.org/research/amigo/default.aspx
(2016). Accessed 1 Sep 2018.

5. Ng CS, Man Chu C, Kwok MW, et al. Hybrid DynaCT scan-guided localization single-port lobectomy. (corrected). Chest 2015;147:e76-8.

6. Ng CS, Yu SC, Lau RW, et al. Hybrid DynaCT-guided electromagnetic navigational bronchoscopic biopsy†. Eur J Cardiothorac Surg 2016;49:187-8.

7. Jin KN, Park CM, Goo JM, et al. Initial experience of percutaneous transthoracic needle biopsy of lung nodules using C-arm cone-beam CT systems. Eur Radiol 2010;20:2108-15.

8. Kostrzewa M, Kara K, Rathmann N, et al. Computed tomography-assisted thoracoscopic surgery: a novel, innovative approach in patients with deep intrapulmonary lesions of unknown malignant status. Invest Radiol 2017;52:374-80.

9. Hsieh MJ, Wen CT, Fang HY, et al. Learning curve of image-guided video-assisted thoracoscopic surgery for small pulmonary nodules: A prospective analysis of 30 initial patients. J Thorac Cardiovasc Surg 2018;155:1825-1832.e1.

10. Kwok YM, Irani FG, Tay KH, et al. Effective dose estimates for cone beam computed tomography in interventional radiology. Eur Radiol 2013;23:3197-204.

11. Chao YK, Pan KT, Wen CT, et al. A comparison of efficacy and safety of preoperative versus intraoperative computed tomography-guided thoracoscopic lung resection. J Thorac Cardiovasc Surg 2018;156:1974-1983.e1.

12. Yu PSY, Man Chu C, Lau RWH, et al. Video-assisted thoracic surgery for tiny pulmonary nodules with realtime image guidance in the hybrid theatre: the initial experience. J Thorac Dis 2018;10:2933-9.

13. Gill RR, Zheng Y, Barlow JS, et al. Image-guided video assisted thoracoscopic surgery (iVATS) - phase I-II clinical trial. J Surg Oncol 2015;112:18-25.

14. Ujiie H, Effat A, Yasufuku K. Image-guided thoracic surgery in the hybrid operation room. J Vis Surg 2017;3:148.

15. $\mathrm{Ng} \mathrm{CSH}$, Chu CM, Lo CK, et al. Hybrid operating room Dyna-computed tomography combined imageguided electromagnetic navigation bronchoscopy dye marking and hookwire localization video-assisted thoracic surgery metastasectomy. Interact Cardiovasc Thorac Surg 2018;26:338-40.

Cite this article as: Zhao ZR, Lau RW, Yu PS, Ng CS. Devising the guidelines: the techniques of pulmonary nodule localization in uniportal video-assisted thoracic surgery—hybrid operating room in the future. J Thorac Dis 2019;11(Suppl 16):S2073-S2078. doi: 10.21037/jtd.2019.01.82 